\title{
DEVELOPMENT OF SOCIAL SYSTEMS IN THE CONTEXT OF PROGNOSIS
}

\author{
Svetlana Kvesko $^{1 *}$, Mikhail Kornienko ${ }^{l}$, Nadezhda Petrunina $^{l}$, Nataliya Kabanova ${ }^{2}$ \\ ${ }^{1}$ National Research Tomsk State University, 634050, Tomsk, Russia \\ ${ }^{2}$ National Research Tomsk Polytechnic University, 634050, Tomsk, Russia
}

\begin{abstract}
The paper dwells on the prognosis of the social systems development. The prognostic analysis is based on the systemic approach to the issue; it shows that the conditions of the transference to the knowledge society determine the formation of the brand new features in social prognoses. The paper states the specific features and conditions of social prognosis, which ensure the validity of control actions. Besides, the current analysis provides recommendations on how to devise social prognostic strategies (within the frame of a) transference to the knowledge society and b) environmental instability). Finally, the functional load of social prognosis in the contemporary conditions is outlined.
\end{abstract}

\section{Introduction}

The process of interacting with such systems is characterized by non-linearity, which lies in the realm of system response, i.e., the system can react to the impact factor disproportionately to the intensity of the latter in accordance with the non-linear law. Besides, social systems, as the open-end ones, involve the incipience of continuous alterations, which, in turn, leads to the high complexity of prognostic schemes.

Multiple ambiguities of the social environment aggravate the management complexity caused by non-linearity of social processes within the frame of transference to the knowledge society, while the potential social systems' development determines the spectral breadth of a potential trajectory, as well as the complexity of problems arising the reform. Within the frame of current circumstances, the dynamic- and tendency-oriented structuring of relevant prognoses becomes one of the hot and the most complicated tasks to perform. The specificity of social systems development in the context of transference to the knowledge society seems significant enough to become the subject of current research.

The possibility of structuring prognostic schemes for the social dynamic pattern has been causing a lot of debate throughout decades. Thus, Karl Popper - being in denial of objective social development regulations - argued that history does not make sense at all whereas any kind of social prognosis can lead to nothing but a mass collapse [1]. Alvin Toffler, American philosopher and social-theorist, in his attempts at systematizing the society's future, anticipates the gigantic changes lying ahead of us. Toffler's futuristic extrapolations concern the exponential sophistication of social systems, the increase in the

*Corresponding author: svetla_kvesko@mail.ru 
multiplicity of developmental alternatives, and the increase in the decision-making complexity $[2,3]$.

One of the first theoretically grounded and significant attempts at social prognosis was made by Daniel Bell in his influential book The Coming of Post-Industrial Society [4]. According to Bell, his work had the purpose of outlining the trends aimed at the delineation of humankind community in XXI century. The theory of post-industrial society made Bell one of the most renowned prognostic theorists in the West; it defines the framework of social research, yet, sets no strict constraints.

The importance of structuring the social system prognostic trajectories remained a subject of interest for a whole bunch of significant thinkers. The modern phase of social development is characterized by the presence of a big number of global threats that evoke the unheard-of risks and dangers for the whole humankind and thus transform the prognostic solutions into improbable matters [5-11].

The socially conscious approach to global threats, as well as to the controversies of the modern world, has been accompanied by the buildup of a specific type of society - that is, the knowledge society predetermined by the development $[12,13]$.

Furthermore, the role of knowledge in the economic phenomena was intensely explored by Joseph Schumpeter [14]. In 1970s Daniel Bell suggested a notion of postindustrial society that centers around scientific knowledge and employs the latter as a base for social transformations [4]. In the contemporary world, the tools and methods used for application of scientific knowledge are changing rapidly $[15,16]$. According to Peter Drucker, knowledge alters the whole function of society as a complex inter-related system $[12,16]$.

It is important to state that - under the conditions of transference to the knowledge society - the task of structuring the long-term social prognoses becomes increasingly complicated. In the XXI century, the current state of global social context is being subject to radical transformations that initiate the rising tide of diverse global risks, global dangers, and global threats, namely, nuclear, chemical, biogenetic, environmental, economic, and existential ones. In the process of such transformations a new social context is emerging where the risk-production is looming large in all the social undertakings. The riskproduction industry undermines the traditional practice aimed at social prognosis and social emergency prevention [17]. The complexity of social prognostic process is aggravated by the fact that prognoses as phenomena are capable of placing significant influence on the real world. Besides, the existent methods appear to be incapable of taking into account the external (i.e., environmental) ambiguity, as well as the behavioral ambiguity of systems per se $[18,19]$.

\section{Development of social systems in the context of prognosis}

Still, the existent models of complex systems are not consistent in providing clear-cut prognosis of the probable outcome due to various ambiguities that reduce the accuracy of the model, namely:

- ambiguity evoked by absence or lack of knowledge for the influential factors / processes;

- ambiguity evoked by incommensurability of numerical estimations for the descriptive values of a system's dynamic pattern;

- ambiguity caused by non-linearity, as well as the presence of numerous equilibrium states and systemic attractors;

- ambiguity evoked by lack of accuracy in the employed vocabulary and consequent inability to render the facts. 
Due to the listed factors, the decision-making that concerns the issue of trajectorial selection is accompanied by a number of problems. Firstly, it is difficult to figure out the sufficient parameters that would allow a predictor to determine the systemic condition and ensure its manageability. The mentioned process bears the name of formalization; the latter, being applied within the realm of social systems - characterized by heterogeneous components (e.g., technological, biological, political, etc.), requires a steady set of parameters in order that the situational context may be properly described. Secondly, information overload is transfiguring into as problem caused by the rising tide of open access information. The tendency to push the limits in the process of considering all the existent parameters and factors leads to information overdose. Any decision that is made loses its importance even before the step due to the low speed of incoming data processing - compared to the speed of environmental and systemic transfigurations. Thirdly, the phenomenon known as emergence, or 'synergetic effect' unfolds another significant problem. The Interacting systems tend to create a higher-level system possessing its own peculiar properties not characteristic of the sub-systems involved. The precise and accurate prognosis based on systemic properties and traits becomes impossible because of the freshly emerged unique supra-system.

The ambiguities described above aggravate the complexity of management process being stipulated by non-linearity of social processes - whereas the potential variance of social systems development determines the wide range of probable spectra, as well as the complexity of problems that tend to occur. This peculiarity was pointed out and described by a number of scholars; thus, Arnold Toynbee highlighted the numerous developmental alternatives typical of human communion at the moment known as bifurcation point, i.e., the turning point transferring the system to the realm of instability [20,21]. Under these circumstances, the exploration of social prognoses and the ways of their outlining becomes one of the hottest and the most complicated tasks that need to be solved in order that social systems may become adaptable to the non-linear environment [22].

L. Zadeh - one of the synergetic paradigm pioneers - formulated the so-called incompatibility principle according to which the system's complexity is directly proportional to the probability of its precise description [23]. However, the postulate of ultimate unpredictability for developmental trajectories under non-linear conditions (suggested by I. Prigogine - yet another prominent pioneer of contemporary synergetics [24]) is lately being widely criticized. We believe that the Kurdiumov's weird attractors scholarship presents a more reliable basis for modeling in social terms [25]. Since the spectrum of a probable outcome is limited, the systemic behavioral pattern cannot be totally unpredictable and is consequently subject to adequate description and subjective anticipation - not in terms of unmistakably predetermined outcome but in terms of belonging to a limited spectrum of multiple conditions.

Transference to the knowledge society currently becomes particularly noteworthy. Knowledge brings changes into behavioral patterns and the whole identity of social environment. High responsiveness and peculiar ambiguity of social environment has long ago become one of the characteristic companions of the global information space. The simultaneous use of information and communication technology (ICT) provided facilities for certain qualitative alterations in the manner of social interaction and adaptation.

Information revolution displays the correlation between ICT and social development process - as it has triggered the formation of macro trends based on the expansion of interactive capacity within social systems. To a considerable extent, the mentioned systems appeared due to the rising integrative processes. Further, the latter catalyzed globalization. Nevertheless, the absence of framed determinism in a social behavioral pattern expands the developmental tracks spectra in the context of forming brand-new social structures. Such structures tend to be characterized as flexible and thus subject to efficient decision-making, 
which, from the viewpoint of the authors, breaks the boundaries of social prognosis and social management.

Within the framework of changeable environment and management troubles, social systems are in desperate need of boosting their flexibility and adaptability, which notably depend on prognostic strategies and sheer probability of anticipating social evolutionary tracks and environmental dynamic pattern.

Growing environmental instability has lately become a crucial factor that predetermines the specificity of the way social systems work and develop. At the same time, it does not simply foster the brand new opportunities - but evokes a bunch of additional problems faced by social systems. One of the chief and central problems is promoting and furthering the adaptability of social systems within the complex and changeable environment. The system can be described as sufficiently adaptable when it is permanently exposed in the light of vital alterations that allow the system to survive and develop whereas the adaptation per se pertains to the process of a system's merge with the new conditions. Traditionally, adaptive systems can be divided into self-adjusting and self-organizing ones. While responding to external alterations, the former change their functional parameters without insystem rearrangements while the latter are able to rearrange their internal structure. For social systems, structural changes can be expressed via modifications in the management frameworks, systemic interrelations, etc.

The adaptable system is able to struggle with the crises caused by its internal dynamic pattern, as well as by the environmental changes. Basically, the social system adaptability predetermines the probable spectrum of its behavioral patterns within unstable environment. There exists a bunch of approaches to the analysis of social adaptability phenomenon. From the standpoint of the first approach, the adaptable system is characterized as single entity and noted for its consistency - for which read, the system's gist it traced throughout all its levels. It seems appropriate to compare an adaptable system to a fractal one where one and the same geometrical pattern is generally reproduced on a reduced scale. Another approach suggests that system be regarded as a living body. In the same way that cells, however different in terms of their functional load, still store all the vital information, the divergent social subsystems contain information about the system and remain under control of the ultimate goal to survive and improve.

Although evident and significant differences between the two mentioned approaches can be easily traced, there is a trait they hold in common: both of them claim unity to be the essence of social system structuring. Unifying factors can be various: the functional load / goal, the accepted type of interconnections and interrelations, the method of information exchange, etc.

Obviously, the social system adaptability is currently provided by numerous factors. The key factor can be traced in the cumulative nature of information processing, in the knowledge transfer, and in the informational navigation within a social system. The most beneficial seem to be the systems that employ the group dynamics method based on the self-organization paradigm. Such systems tend to be highly adaptable thanks to their capability to generate, select, and store novelties and significant alterations - thus providing permanent self-upgrade.

As noted by Ikujito Nonaka [26], the essence of upgrade processing can be presented as a sequence of four steps:

- emergence of chaos / instability within a system;

- chaotic aggravation and controversy focusing;

- emergence of self-organized subsystems and interaction adjustments;

- $\quad$ knowledge transference.

We believe that ability to coin novelties is a primary sign of a system's diversity - as was formulated by William Ashby in An Introduction to Cybernetics [27]: "In order to 
create the system capable of solving a diverse task, we need to make the system's diversity wider than that of the task - or to ensure that the system has the potential to generate it." In case of social system that implies that under the influence of multiple external factors a system is able to succeed in the adaption process only providing the expansion of control modifiers. Still, the close analysis of various systems demonstrates that their sustainability, adaptability, and improvement potential are directly proportional to the degree of systemic diversity. The sequential changes in the social self-renovation process are nothing but a mechanism of diversity boost oriented towards the expansion of a system's capacity to resolve the adaptability issues.

\section{Conclusion}

Vast number of adaption issues in the realm of social systems is stipulated by the complexity of the dynamic pattern characterized by the following traits:

- evolutionary development;

- instability;

- self-management and self-reproduction;

- procedural asymmetry.

Nevertheless, it is not the external factors that determine the social adaptation outcome - but their internal dynamic pattern, which is primarily predetermined by the system ergodicity. The presence of immanent aims and criteria in the functional and developmental scopes of social systems allows us to argue that the internal interactions play the key role in sketching the direction of evolutionary process.

The multiplicity of choices at bifurcation points (being a crucial feature of non-linear complex systems) increases due to social systems, which is predetermined by the fact that informational, cultural, and psychological correlations prevail over physical interactions. What is more, the multiplicity of systemic, changeable, and subjective aims / criteria elicits additional changeability and thus leads to the predominance of the unpredictable over the anticipated. The listed peculiarities ground the necessity to maintain social systems on the level of sufficient flexibility and adaptability - to ensure the functioning of the latter within unstable conditions.

Globalization brought on changes in social structure and functional load; social systems nowadays are getting increasingly flexible and sensitive to any kind of external alteration. Systemic evolution is permanently accompanied by the increase in complexity and necessity to adapt to the open-end, ever-transforming environment. For social system that stipulates the rising significance of innovative enterprises. We argue that sustainability of social systems is directly linked to innovations based on co-adaptation and co-evolution.

In this particular case co-adaptation can be expressed as equilibrium in the assimilation and accommodation within social systems, as well as systemic mutual adaptation. What is more, the identity of social adaptation processes is undergoing a number of changes; social systems tend to replace genesis (i.e., adaptation to the given condition) with environmental enhancement. This tendency displays itself via diversity of:

a) flexible and changeable interconnections;

b) ways of mutual coordination;

c) high heterogeneity of internal structure;

d) self-regeneration and self-reproduction.

Thus, co-adaptation provides the context for co-evolution that involves the sequence of coordinate, indissolubly adjusted alterations capable of taking place at any evolutionary level of a social system. 
Consequently, the increasing management complexity in the realm of modern social systems is determined by their specificity - on the one hand. On the other hand, there goes another factor, namely, the high speed of social dynamic pattern changeability predetermined by the transference to the knowledge society placing restrictions on forecasting horizon and on allowed time of control actions. In contrast, the mentioned processes stipulate the necessity to figure out effective prognostic-based methods of managing social systems that would speed up the adaption to the unstable environment. Self-organization procedure provides the optimal directions in the process of managing social systems.

Interaction with such systems implies non-linearity displayed by the level of system response to contributing factor, which is not proportionate to the intensity of the latter but complies with the non-linear law. The base for self-fulfillment or self-destruction of a prophecy lies in the frame of positive vs. negative public reaction.

In conclusion, whatever social prognostic trajectory may be, it is always hypothetical, i.e., it concerns mainly the developmental trends, not the actual future states of a social system. Within the frame of transference to the knowledge society, due to multiplicity of a probable outcome, because of the prognosis instability with regard to both external and internal impact an essential part of social prognoses is ruled out invalid. Nevertheless, even invalid prognoses carry a significant functional load: any social prognosis, be it valid or not, reveals the probable multiplicity of social developmental alternatives, allows to evaluate the consequences of decision-making process, and serves as a warning of high risks and disastrous developmental tracks.

\section{References}

[1] K. Popper, The Open Society and Its Enemies (Feniks, Moscow, 2015)

[2] A. Toffler, The Third Wave (William Morrow, NewYork, 2014)

[3] S. Kvesko, T. Shinn, MATEC Web of Conf. 79, 01048 (2016)

[4] D. Bell, The Coming of Post-Industrial Society. A Venture in Social Forecasting (Basic Books, New York, 2013)

[5] E. Vasendina, I. Plotnikova, A. Levitskaya, S. Kvesko, IOP Conf. Series: Mater. Sc. and Engin. 110, 012070 (2016) doi:10.1088/1757-899X/110/1/012070

[6] A. Toffler, Future Shock (Bantam Books, New York, 2002)

[7] U. Beck, Risk Society (Toward a New Modernity, London: SAGE. 1992)

[8] M. Castells. The Rise of the Network Society (Blakwell Publishers, London, 2010)

[9] A. A. Kornienko. Proc. Soc. and Behav. Sc. 166 (2015)

[10] F. Janszen. The Age of Innovation (Prentice Hall, London, 2000)

[11] V. I. Syryamkin, D. S Zhdanov. Telecomm. and Radio Engin. (2012)

[12] P. Drucker, Post-capitalist Society (HarperCollins, New York, 1993)

[13] F. Machlup, The Production and Distribution of Knowledge in the United States. (Princeton University Press, Princeton, 1962)

[14] J. Schumpeter, Theory of Economic Development (Progress, Moscow, 2012)

[15] V. Bestuzhev-LadaI, Prognostic Grounding of Social Innovations (Academia, Moscow, 2011)

[16] P. Drucker, Innovation and Entrepreneurship (Collins, New York, 2012)

[17] A. Tolstoukhov, Issues of Philosophy 8, 49 (2003)

[18] A. V. Yurchenko, MATEC Web of Conf. 79, (2016)

[19] N. V. Chicherina, V. Kazakov, O. Yu. Korneva, The European Proceedings of Social \& Behavioural Sciences (EpSBS) 19, 349 (2017) doi: 10.15405/epsbs.2017.01.47

[20] N. V. Chicherina, V. A. Tolkacheva, The European Proceedings of Social \& Behavioural Sciences (EpSBS) 19, 683 (2017) doi:10.15405/epsbs.2017.01.90 
[21] A. J. Toynbee, A Study of History (Progress, Moscow 2011)

[22] S. B. Kvesko, S. E. Kvesko, N. Kabanova, D. P. Shamrova, MATEC Web of Conf. 79, 01062 (2016)

[23] L. A. Zadeh, IEEE Trans. Syst. Man. Cybern. 1, 28. (1973)

[24] I. Prigogine, I. Stengers, Order out of Chaos. Man's New Dialogue (Progress, Moscow, 2014)

[25] S. P. Kurdiumov, E. N. Knyazeva, Laws of Evolution and Self-Organization in Complex Systems (Nauka, Moscow, 2015)

[26] I. Nonaka, Creating Organizational Order out of Chaos: Self-renewal in Japanese Firms (University Press, New York, 2008)

[27] W. R. Ashby, Introduction to Cybernetics (Nauka, Moscow, 2014) 